\title{
Isothiazolopyridine Mannich bases and their antibacterial effect
}

\author{
Piotr Świątek ${ }^{A-F}$, Małgorzata Strzelecka ${ }^{B, C}$ \\ Department of Chemistry of Drugs, Wroclaw Medical University, Poland \\ A - research concept and design; $\mathrm{B}$ - collection and/or assembly of data; $\mathrm{C}$ - data analysis and interpretation; \\ $\mathrm{D}$ - writing the article; $\mathrm{E}$ - critical revision of the article; $\mathrm{F}$ - final approval of the article
}

\section{Address for correspondence \\ Piotr Świątek}

E-mail: piotr.swiatek@umed.wroc.pl

\section{Funding sources}

This research was supported by Wroclaw Medical University (project No. STD 07017026).

\section{Conflict of interest}

None declared

\section{Acknowledgements}

The authors thank the Community for Open Antimicrobial Drug Discovery, Institute for Molecular Bioscience, University of Queensland, Brisbane, Australia for carrying out antibacterial assays.

Received on June 13, 2018 Reviewed on July 16,2018 Accepted on October 30, 2018

Published online on December 18, 2018

\begin{abstract}
Background. Infections caused by multidrug-resistant (MDR) strains, i.e., strains resistant to at least 1 antibiotic of the 3 groups of antibacterial agents, are among the most difficult to treat. New compounds with an antimicrobial action are being sought in order to avoid the complete resistance of bacteria to drugs and the spread of MDR strains.

Objectives. The aim of the research was to determine the antimicrobial activity of the new isothiazolopyridine derivatives.

Material and methods. All chemicals used were purchased from commercial suppliers. The ${ }^{1} H$ NMR spectra were recorded on a Bruker $300 \mathrm{MHz}$ NMR spectrometer. Infrared (IR) spectra were run on a Perkin-Elmer Spectrum Two UATR FT-IR spectrometer (Perkin-Elmer, Waltham, USA). Elemental analyses were carried out on a Carlo Erba NA 1500 analyzer (Carlo Erba Reagents SAS, Val de Reuil, France). Melting points were determined with a Mel-Temp II apparatus (Laboratory Devices, Holliston, USA). The bacteria panel, including Escherichia coli ATCC 25922, Klebsiella pneumoniae ATCC 700603 (MDR), Acinetobacter baumannii ATCC 19606, Pseudomonas aeruginosa ATCC 27853, and multidrug-resistant Staphylococcus aureus ATCC 43300 (MRSA) were cultured in Muller-Hinton broth (MHB) at $37^{\circ} \mathrm{C}$ overnight. Colistin, Polymyxin B, Vancomycin, and Daptomycin were used as controls of bacterial inhibitors. Inhibition of bacterial growth was determined visually and was recorded at $32 \mu \mathrm{g} / \mathrm{mL} ; 100 \%$ inhibition was identified.
\end{abstract}

Results. The new dimethylisothiazolopyridines were prepared by the Mannich reaction. The structures of the isothiazolopyridines were determined based on spectral data analysis, such as IR and ${ }^{1} H$ NMR. The antimicrobial screening of new compounds was performed. In the primary screen, 2 compounds showed antimicrobial activity (minimum inhibitory concentration (MIC) $\leq 32 \mu \mathrm{g} / \mathrm{mL}$ ).

Conclusions. Taking into account the obtained results, it should be stated that the examined compounds did not exceed the activity of reference drugs and, therefore, further research should be carried out in the group of isothiazolopyridine derivatives.

Key words: synthesis, antibacterial agents, isothiazolopyridine

\section{Cite as}

Świątek P, Strzelecka M. Isothiazolopyridine Mannich bases and their antibacterial effect. Adv Clin Exp Med. 2019;28(7):967-972. doi:10.17219/acem/99310

DOI

10.17219/acem/99310

\section{Copyright}

Copyright by Author(s)

This is an article distributed under the terms of the Creative Commons Attribution Non-Commercial License (http://creativecommons.org/licenses/by-nc-nd/4.0/) 
Despite significant progress in the diagnosis and treatment of infectious diseases, bacterial infections continue to pose a serious threat to human life. According to a report published in 2011 by the World Health Organization, diseases caused by bacteria are one of the leading causes of death. Naturally occurring compounds and compounds obtained with chemical synthesis, which show bacterial growth inhibitory effect (bacteriostatic agents) or cause the death of bacteria (antimicrobial substances), are used to counter infections.

Unfortunately, the problem of drug resistance is growing along with the development of new antibacterial agents. This phenomenon no longer concerns only nosocomial pathogens, but increasingly also non-nosocomial pathogens. Infections caused by multidrug-resistant (MDR) strains, i.e., strains resistant to at least 1 antibiotic of the 3 groups of antibacterial agents, are among the most difficult to treat. In addition, extensively drug-resistant (XDR) strains have emerged, i.e., strains resistant to only 1 antibiotic of 1 or 2 groups of drugs used in the treatment of infections caused by this microorganism.

Some microorganisms are resistant to all available antibiotics and can only be countered with the use of experimental and often potentially toxic drugs - these are the so-called pandrug-resistant (PDR) strains. ${ }^{1}$

The limited possibility of effective treatment of infections results in increased morbidity and mortality due to bacterial infections. This is reflected by the data contained in a report prepared in 2009 by the European Medicines Agency (EMA) and the European Centre for Disease Prevention and Control (ECDC). They reveal that MDR strains cause approx. 25,000 deaths per year throughout the European Union.

The constantly increasing number of infections caused by MDR strains and a decrease in the number of new antibiotics introduced into medical practice may send us back to the "pre-antibiotics era". ${ }^{2}$ New compounds with an antimicrobial action are being sought in order to avoid the complete resistance of bacteria to drugs and the spread of MDR strains.

The new antibacterial agents are developed by modifying existing antibacterial drugs or by creating a new class of drugs. Among a large number of new classes of compounds synthesized for this purpose, derivatives of bicyclic structures (benzoxazole, benzothiazole, benzisothiazole, quinolone, qinoxaline) exhibited promising results. ${ }^{3-9}$
In particular, benzisothiozol-3(2H)-one derivatives have a wide range of antimicrobial activity $y^{10,11}$ and have been reported to react with thiol-containing proteins on target microorganisms, and are particularly potent against actively metabolizing cells. ${ }^{12,13}$

Taking into account the interesting antibacterial activity of benzisothiazoles, 4,6-dimethylisothiazolo[5,4-b] pyridine derivatives were synthesized. They can be treated as 7-aza analogues of benzisothiazoles. The new compounds were designed as N-Mannich bases with piperazine or piperidine residues in the side chain. Some authors suggest that such substituents may play the role of a pharmacophore for antibacterial activity. ${ }^{14,15}$

\section{Material and methods}

\section{Chemistry \\ Chemical experimental section}

All chemicals used were purchased from commercial suppliers. A dry solvent was obtained according to the standard procedure. The progress of the reaction was monitored by TLC on silica gel 60 F254-coated TLC plates (Fluka Chemie GmbH, Buchs, Switzerland) and visualized by UV light at $254 \mathrm{~nm}$. The proton nuclear magnetic resonance ( ${ }^{1} \mathrm{H}$ NMR) spectra were recorded on a Bruker $300 \mathrm{MHz}$ NMR spectrometer (Bruker, Billerica, USA) in $d$-chloroform $\left(\mathrm{CDCl}_{3}\right)$, while tetramethylsilane (TMS) was used as an internal reference. Chemical shifts are given in ppm units, and coupling constant values are given in $\mathrm{Hz}$. Infrared (IR) spectra were run on a Perkin-Elmer Spectrum Two UATR FT-IR spectrometer UATR - universal attenuated total reflection; Perkin-Elmer, Waltham, USA), and frequencies are reported in $\mathrm{cm}^{-1}$. The samples were applied as solids. Elemental analyses for carbon, nitrogen and hydrogen were carried out on a Carlo Erba NA 1500 analyzer (Carlo Erba Reagents SAS, Val-de-Reuil, France) and were within $\pm 0.4 \%$ of the theoretical value. Melting points were determined with a Mel-Temp II apparatus (Laboratory Devices, Holliston, USA) and were uncorrected.

General procedure for the preparation of $6,7,9,11,12,15$, and 16

A mixture of $2.5 \mathrm{mmol}$ of 4,6-dimethylisothiazolo[5,4-b]pyridin-3(2H)-one 1 with $0.5 \mathrm{~mL}$ of formaline (37\% water solution) and $2.5 \mathrm{mmol}$ of corresponding amine (piperazine or piperidine) derivatives in $30 \mathrm{~mL}$ of THF was refluxed with stirring for 10-11 h. After this time, the mixture was evaporated to dryness. The crude product was then purified by crystallization from appropriate solvents.

1. 4,6-dimethyl-2-[4-(4-iodophenyl)piperazin-1-yl-methyl]isothiazolo[5,4-b]pyridin-3(2H)-one 6

Anal. $\mathrm{C}_{19} \mathrm{H}_{21} \mathrm{IN}_{4} \mathrm{OS}$ (m.w. 480.36); 55\% yield, m.p. 172-174 ${ }^{\circ} \mathrm{C}$ (cyclohexane), FT-IR (UATR, selected lines) $v_{\max } / \mathrm{cm}^{-1}$ : $1680(\mathrm{C}=\mathrm{O}),{ }^{1} \mathrm{H}$ NMR $\left(\mathrm{CDCl}_{3}\right) \delta: 2.60\left(\mathrm{~s}, 3 \mathrm{H}, \mathrm{CH}_{3-\text { pyridine}}\right), 2.74\left(\mathrm{~s}, 3 \mathrm{H}, \mathrm{CH}_{3}{ }^{-}\right.$pyridine$), 2.84-2.88\left(\mathrm{~m}, 4 \mathrm{H}, 2 \times \mathrm{CH}_{2}\right.$-piperazine), 3.17-3.21 (m, 4H, $2 \times \mathrm{CH}_{2}$-piperazine $) 4.71\left(\mathrm{~s}, 2 \mathrm{H}, \mathrm{CH}_{2}\right), 6.65-6.68(\mathrm{~m}, 2 \mathrm{H}, \mathrm{PhH}), 6.94\left(\mathrm{~s}, 1 \mathrm{H}, \mathrm{H}_{\beta-p y r i d i n e}\right), 7.49-7.52(\mathrm{~m}, 2 \mathrm{H}, \mathrm{PhH})$ 
2. 4,6-dimethyl-2-[4-(2-cyanophenyl)piperazin-1-yl-methyl]isothiazolo[5,4-b]pyridin-3(2H)-one 7

Anal. $\mathrm{C}_{20} \mathrm{H}_{21} \mathrm{~N}_{5} \mathrm{OS}$ (m.w. 379.47); $96 \%$ yield, m.p. $161-163^{\circ} \mathrm{C}$ (ethyl acetate), FT-IR (UATR, selected lines) $v_{\max } / \mathrm{cm}^{-1}: 2220$

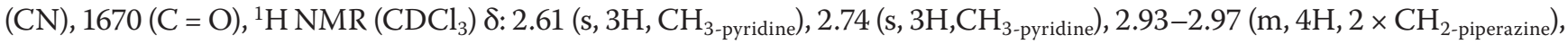

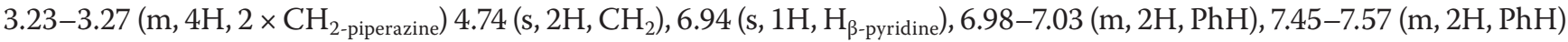

3. 4,6-dimethyl-2-[4-ethyl-2,3-dioxopiperazin-1-yl-methyl]isothiazolo[5,4-b]pyridin-3(2H)-one 9

Anal. $\mathrm{C}_{15} \mathrm{H}_{18} \mathrm{~N}_{4} \mathrm{O}_{3} \mathrm{~S}$ (m.w. 334.39); $47 \%$ yield, m.p. $147-149^{\circ} \mathrm{C}$ (ethyl acetate), FT-IR (UATR, selected lines) $v_{\max } / \mathrm{cm}^{-1}$ : $1670(\mathrm{C}=\mathrm{O}),{ }^{1} \mathrm{H}$ NMR $\left(\mathrm{CDCl}_{3}\right) \delta: 1.20\left(\mathrm{t}, 3 \mathrm{H}, \mathrm{CH}_{3}, \mathrm{~J}=9 \mathrm{~Hz}\right) 2.59$ (s, 3H, $\left.\mathrm{CH}_{3 \text {-pyridine }}\right), 2.71$ (s, 3H, $\left.\mathrm{CH}_{3 \text {-pyridine }}\right), 3.49-3.59$

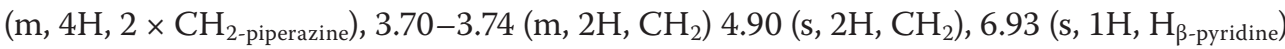

4. 4,6-dimethyl-2-[4-(tetrahydrofuran-2-yl-carbonyl)piperazin-1-yl-methyl]isothiazolo[5,4-b]pyridin-3(2H)-one 11

Anal. $\mathrm{C}_{18} \mathrm{H}_{24} \mathrm{~N}_{4} \mathrm{O}_{3} \mathrm{~S}$ (m.w. 376.47); $42 \%$ yield, m.p. $126-128^{\circ} \mathrm{C}$ (cyclohexane), FT-IR (UATR, selected lines) $v_{\max } / \mathrm{cm}^{-1}$ : $1680(\mathrm{C}=\mathrm{O}), 1650(\mathrm{C}=\mathrm{O}),{ }^{1} \mathrm{H}$ NMR $\left(\mathrm{CDCl}_{3}\right) \delta: 1.92-2.06\left(\mathrm{~m}, 4 \mathrm{H}, \mathrm{CH}_{2}\right), 2.59\left(\mathrm{~s}, 3 \mathrm{H}, \mathrm{CH}_{3 \text {-pyridine }}\right), 2.68-2.72(\mathrm{~m}, 7 \mathrm{H}$, $2 \times \mathrm{CH}_{2 \text {-piperazine }}$ and $\left.\mathrm{CH}_{3 \text {-pyridine }}\right), 3.53-3.69\left(\mathrm{~m}, 4 \mathrm{H}, 2 \times \mathrm{CH}_{\text {2-piperazine }}\right), 3.77-3.94\left(\mathrm{~m}, 2 \mathrm{H}, \mathrm{CH}_{2}\right), 4.54-4.58(\mathrm{~m}, 1 \mathrm{H}, \mathrm{CH}) 4.68$ (s, 2H, $\left.\mathrm{CH}_{2}\right), 6.93$ (s, $\left.1 \mathrm{H}, \mathrm{H}_{\beta \text {-pyridine }}\right)$

5. 4,6-dimethyl-2-[(4-methyl-2-phenylpiperazin-1-yl)methyl]isothiazolo[5,4-b]pyridin-3(2H)-one 12

Anal. $\mathrm{C}_{20} \mathrm{H}_{24} \mathrm{~N}_{4} \mathrm{OS}$ (m.w. 368.49); $27 \%$ yield, m.p. $108-112^{\circ} \mathrm{C}$ (cyclohexane), FT-IR (UATR, selected lines) $v_{\max } / \mathrm{cm}^{-1}$ : $1675(\mathrm{C}=\mathrm{O}),{ }^{1} \mathrm{H}$ NMR $\left(\mathrm{CDCl}_{3}\right) \delta: 2.27$ (s, 3H, $\left.\mathrm{CH}_{3}\right), 2.58$ (s, 3H, $\left.\mathrm{CH}_{3 \text {-pyridine }}\right), 2.69$ (s, 3H, $\mathrm{CH}_{3 \text {-pyridine) }}, 2.73-2.85$ (m, 3H, $\mathrm{CH}$ and $\mathrm{CH}_{2 \text {-piperazine) }}$, 3.14-3.18 (m, $2 \mathrm{H}, \mathrm{CH}_{\text {2-piperazine) }}$, 3.74-3.78 (m, 2H, $\mathrm{CH}_{2 \text {-piperazine) }}$ ) 4.67 (s, $\left.2 \mathrm{H}, \mathrm{CH}_{2}\right), 6.89$ (s, $1 \mathrm{H}$, $\mathrm{H}_{\beta-\text { pyridine }}$ ), 7.31-7.40 (m, 3H, PhH), 7.49-7.52 (m, 2H, PhH)

6. 4,6-dimethyl-2-[((4-piperidin)piperidin-1-yl)methyl]isothiazolo[5,4-b]pyridin-3(2H)-one 15

Anal. $\mathrm{C}_{19} \mathrm{H}_{28} \mathrm{~N}_{4} \mathrm{OS}$ (m.w. 360.51); 74\% yield, m.p. $162-164^{\circ} \mathrm{C}$ (ethyl acetate), FT-IR (UATR, selected lines) $v_{\max } / \mathrm{cm}^{-1}$ : $1670(\mathrm{C}=\mathrm{O}),{ }^{1} \mathrm{H}$ NMR $\left(\mathrm{CDCl}_{3}\right) \delta: 1.40-1.42\left(\mathrm{~m}, 2 \mathrm{H}, \mathrm{CH}_{2 \text {-piperidine }}\right), 1.52-1.60\left(\mathrm{~m}, 5 \mathrm{H}, 2 \times \mathrm{CH}_{2 \text {-piperidine }}\right.$ and $\left.\mathrm{CH}\right), 1.78-1.83$

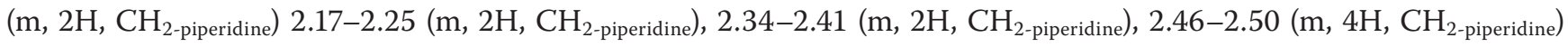
2.59 (s, 3H, $\mathrm{CH}_{3 \text {-pyridine) }}, 2.72\left(\mathrm{~s}, 3 \mathrm{H}, \mathrm{CH}_{3 \text {-pyridine }}\right), 3.04-3.09\left(\mathrm{~m}, 2 \mathrm{H}, \mathrm{CH}_{2 \text {-piperidine }}\right), 4.64\left(\mathrm{~s}, 2 \mathrm{H}, \mathrm{CH}_{2}\right), 6.91\left(\mathrm{~s}, 1 \mathrm{H}, \mathrm{H}_{\beta}\right.$-pyridine $)$

7. 4,6-dimethyl-2-[((4-benzyl-4-hydroxy)piperidin-1-yl)methyl]isothiazolo[5,4-b]pyridin-3(2H)-one 16

Anal. $\mathrm{C}_{21} \mathrm{H}_{25} \mathrm{~N}_{3} \mathrm{O}_{2} \mathrm{~S}$ (m.w. 383.50); 63\% yield, m.p. $106-108^{\circ} \mathrm{C}$ (cyclohexane), FT-IR (UATR, selected lines) $v_{\max } / \mathrm{cm}^{-1}$ :

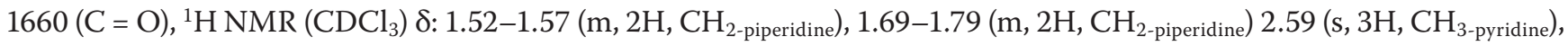

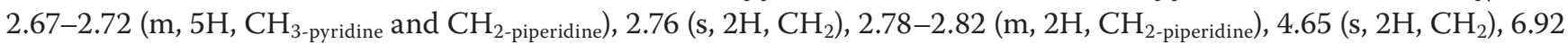
(s, 1H, $\mathrm{H}_{\beta-\text {-pyridine) }}$, 7.18-7.21 (m, 2H, PhH), 7.24-7.33 (m, 3H, PhH)

\section{General procedure for the preparation of 3, 4, 5, 10, 13 and 14}

To a stirred mixture of $2.4 \mathrm{mmol}$ of 2-hydroxymethyl-4,6-dimethylisothiazolo[5,4-b]pyridin-3(2H)-one 2 in $20 \mathrm{~mL}$ of ethanol, $2.4 \mathrm{mmol}$ of appropriate amine was added and the stirring was continued for $24 \mathrm{~h}$ at room temperature. Then the precipitated crude product was filtered off and crystallized from the appropriate solvent.

1. 4,6-dimethyl-2-[(4-(2,3-dichlorophenyl)piperazin-1-yl)methyl]isothiazolo[5,4-b]pyridin-3(2H)-one 3

Anal. $\mathrm{C}_{19} \mathrm{H}_{20} \mathrm{Cl}_{2} \mathrm{~N}_{4} \mathrm{OS}$ (m.w. 423.36); 74\% yield, m.p. $159-161^{\circ} \mathrm{C}$ (cyclohexane), FT-IR (UATR, selected lines) $v_{\max } / \mathrm{cm}^{-1}$ :

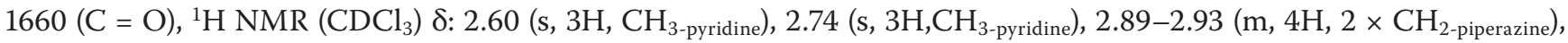

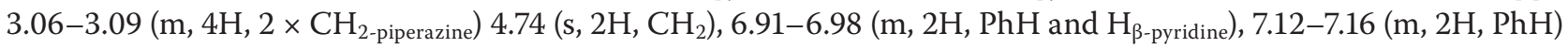

2. 4,6-dimethyl-2-[(4-(3,4-dichlorophenyl)piperazin-1-yl)methyl]isothiazolo[5,4-b]pyridin-3(2H)-one 4

Anal. $\mathrm{C}_{19} \mathrm{H}_{20} \mathrm{Cl}_{2} \mathrm{~N}_{4} \mathrm{OS}$ (m.w. 423.36); $65 \%$ yield, m.p. $146-148^{\circ} \mathrm{C}$ (cyclohexane), FT-IR (UATR, selected lines) $v_{\max } / \mathrm{cm}^{-1}$ :

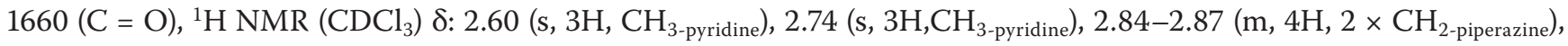
3.17-3.20 (m, 4H, $2 \times \mathrm{CH}_{2 \text {-piperazine })} 4.71\left(\mathrm{~s}, 2 \mathrm{H}, \mathrm{CH}_{2}\right), 6.70-7.74(\mathrm{~m}, 2 \mathrm{H}, \mathrm{PhH})$ 6.91-6.94 (m, 2H, $\mathrm{PhH}$, and $\left.\mathrm{H}_{\beta-\text { pyridine }}\right)$,

3. 4,6-dimethyl-2-[(4-(2,4-difluorophenyl)piperazin-1-yl)methyl]isothiazolo[5,4-b]pyridin-3(2H)-one 5

Anal. $\mathrm{C}_{19} \mathrm{H}_{20} \mathrm{~F}_{2} \mathrm{~N}_{4} \mathrm{OS}$ (m.w. 390.45); 79\% yield, m.p. 130-131 ${ }^{\circ} \mathrm{C}$ (cyclohexane), FT-IR (UATR, selected lines) $v_{\max } / \mathrm{cm}^{-1}$ : 1675 ( $\mathrm{C}=\mathrm{O}$ ), ${ }^{1} \mathrm{H} \mathrm{NMR}\left(\mathrm{CDCl}_{3}\right)$ 8: 2.59 (s, 3H, $\mathrm{CH}_{3 \text {-pyridine }}$ ), 2.73 (s, 3H, $\left.\mathrm{CH}_{3 \text {-pyridine }}\right), 2.87-2.91\left(\mathrm{~m}, 4 \mathrm{H}, 2 \times \mathrm{CH}_{2 \text {-piperazine }}\right.$ ), 3.03-3.06 (m, 4H, $2 \times \mathrm{CH}_{2 \text {-piperazine })} 4.71$ (s, $\left.2 \mathrm{H}, \mathrm{CH}_{2}\right), 6.74-6.82(\mathrm{~m}, 3 \mathrm{H}, \mathrm{PhH}), 6.85-6.91$ (m, 2H, PhH), 6.93 (s, 1H, $\left.\mathrm{H}_{\beta \text {-pyridine }}\right)$ 
4. 4,6-dimethyl-2-[4-cyclohexylcarbonyl-piperazin-1-yl-methyl]isothiazolo[5,4-b]pyridin-3(2H)-one 10

Anal. $\mathrm{C}_{20} \mathrm{H}_{28} \mathrm{~N}_{4} \mathrm{O}_{2} \mathrm{~S}$ (m.w. 388.52); 39\% yield, m.p. 125-128 ${ }^{\circ} \mathrm{C}$ (cyclohexane), FT-IR (UATR, selected lines) $v_{\text {max }} / \mathrm{cm}^{-1}$ : $1680(\mathrm{C}=\mathrm{O}), 1650(\mathrm{C}=\mathrm{O}),{ }^{1} \mathrm{H}$ NMR $\left(\mathrm{CDCl}_{3}\right) \delta: 1.21-1.25\left(\mathrm{~m}, 4 \mathrm{H}, \mathrm{CH}_{2}\right), 1.43-1.51\left(\mathrm{~m}, 2 \mathrm{H}, \mathrm{CH}_{2}\right), 1.64-1.67\left(\mathrm{~m}, 2 \mathrm{H}, \mathrm{CH}_{2}\right)$, 1.75-1.78 (m, 2H, CH

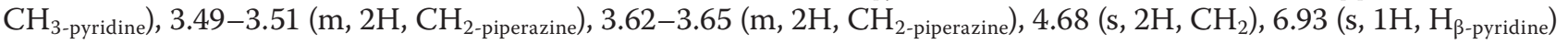

5. 4,6-dimethyl-2-[(4-n-hexylpiperazin-1-yl)methyl]isothiazolo[5,4-b]pyridin-3(2H)-one 13

Anal. $\mathrm{C}_{19} \mathrm{H}_{30} \mathrm{~N}_{4} \mathrm{OS}$ (m.w. 362.53); 74.6\% yield, m.p. 78-80 ${ }^{\circ} \mathrm{C}$ (n-heptane), FT-IR (UATR, selected lines) $v_{\max } / \mathrm{cm}^{-1}: 1650$ $(\mathrm{C}=\mathrm{O}),{ }^{1} \mathrm{H} \mathrm{NMR}_{\left(\mathrm{CDCl}_{3}\right)}$ ): $0.85\left(\mathrm{t}, 3 \mathrm{H}, \mathrm{CH}_{3}, \mathrm{~J}=6 \mathrm{~Hz}\right), 1.21-1.29\left(\mathrm{~m}, 6 \mathrm{H}, \mathrm{CH}_{2}\right), 1.43-1.52\left(\mathrm{~m}, 2 \mathrm{H}, \mathrm{CH}_{2}\right), 2.29-2.35(\mathrm{~m}, 2 \mathrm{H}$,

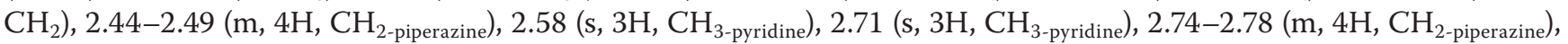
4.64 (s, 2H, $\left.\mathrm{CH}_{2}\right), 6.91\left(\mathrm{~s}, 1 \mathrm{H}, \mathrm{H}_{\beta \text {-pyridine }}\right)$

6. 4,6-dimethyl-2-[(4-phenylpiperidin-1-yl)methyl]isothiazolo[5,4-b]pyridin-3(2H)-one 14

Anal. $\mathrm{C}_{20} \mathrm{H}_{23} \mathrm{~N}_{3} \mathrm{OS}$ (m.w. 353.48); 80\% yield, m.p. 136-138 ${ }^{\circ} \mathrm{C}$ (n-heptane), FT-IR (UATR, selected lines) $v_{\text {max }} / \mathrm{cm}^{-1}: 1670$

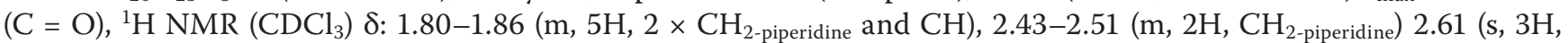

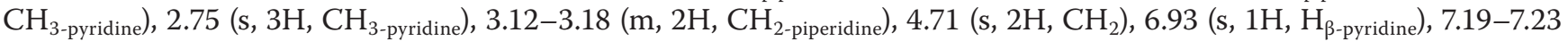
(m, 2H, PhH), 7.27-7.32 (m, 3H, PhH)

\section{General procedure for the preparation of 17 and 18}

To a solution of $0.01 \mathrm{~mol}$ of 4,6-dimethylisothiazolo[5,4-b]pyridin-3(2H)-one 1 in $20 \mathrm{~mL}$ of ethanol, $0.8 \mathrm{~mL}$ of formaline (37\% water solution) was added and the mixture was refluxed for $15 \mathrm{~min}$. After this time, the mixture was cooled, and $0.005 \mathrm{~mol}$ of piperazine or ethylenediamine was added to the precipitated product 2 . Next, the mixture was refluxed for $1 \mathrm{~h}$. After cooling, the precipitated crude product 17 and 18 was filtered off and crystallized from the ethanol.

1. 1,4-bis(4,6-dimethyl-3-oxo-2,3-dihydroisothiazolo[5,4-b]pyridin-2-ylmethyl)piperazine 17

Anal. $\mathrm{C}_{22} \mathrm{H}_{26} \mathrm{~N}_{6} \mathrm{O}_{2} \mathrm{~S}_{2}$ (m.w. 470.60); 44\% yield, m.p. 233-235 ${ }^{\circ} \mathrm{C}$, FT-IR (UATR, selected lines) $v_{\max } / \mathrm{cm}^{-1}: 1680(\mathrm{C}=\mathrm{O}$ ),

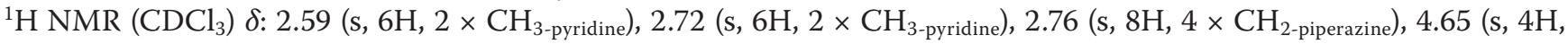
$\left.2 \times \mathrm{CH}_{2}\right), 6.92\left(\mathrm{~s}, 2 \mathrm{H}, 2 \times \mathrm{H}_{\beta \text {-pyridine }}\right)$

2. 1,2-bis(4,6-dimethyl-3-oxo-2,3-dihydroisothiazolo[5,4-b]pyridin-2-ylmethyl)ethylenediamine 18

Anal. $\mathrm{C}_{20} \mathrm{H}_{24} \mathrm{~N}_{6} \mathrm{O}_{2} \mathrm{~S}_{2}$ (m.w. 443.58); $42 \%$ yield, m.p. $148-150^{\circ} \mathrm{C}$, FT-IR (UATR, selected lines) $v_{\text {max }} / \mathrm{cm}^{-1}: 3450-3400$

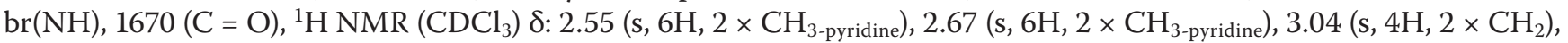
$3.81(\mathrm{~s}, 2 \mathrm{H}, 2 \times \mathrm{NH}), 4.74\left(\mathrm{~s}, 4 \mathrm{H}, 2 \times \mathrm{CH}_{2}\right), 6.88\left(\mathrm{~s}, 2 \mathrm{H}, 2 \times \mathrm{H}_{\beta \text {-pyridine }}\right)$

\section{Pharmacology}

The antimicrobial screening of new compounds was performed by CO-ADD (Community for Open Antimicrobial Drug Discovery, Institute for Molecular Bioscience, University of Queensland, Brisbane, Australia). ${ }^{16}$ Isothiazolopyridines 3-18 were tested for bacterial growth inhibitory effect against a primary panel including Escherichia coli, Klebsiella pneumoniae, Acinetobacter baumannii, Pseudomonas aeruginosa, and multidrugresistant Staphylococcus aureus (MRSA) at a single point concentration of $32 \mu \mathrm{g} / \mathrm{mL}$ and MICs determined for the "hit" compounds from the primary screen. Colistin and Polymyxin B were used as positive inhibitor controls for Gramnegative bacteria. Vancomycin and Daptomycin were used as positive inhibitor controls for Gram-positive bacteria.

\section{Single point bacterial inhibition assay}

The primary bacteria panel, including E. coli ATCC 25922, K. pneumoniae ATCC 700603 (MDR), A. baumannii ATCC 19606, $P$. aeruginosa ATCC 27853, and S. aureus ATCC 43300 (MRSA) were cultured in Muller-Hinton broth
(MHB) at $37^{\circ} \mathrm{C}$ overnight. A sample of each culture was then diluted 40 -fold in fresh $\mathrm{MHB}$ and incubated at $37^{\circ} \mathrm{C}$ for $1.5-3 \mathrm{~h}$. The compounds were plated at a test concentration of $64 \mu \mathrm{g} / \mathrm{mL}$. Colistin, Polymyxin B, Vancomycin, and Daptomycin were serially diluted twice across the wells, with compound concentrations ranging from $0.03 \mu \mathrm{g} / \mathrm{mL}$ to $0.64 \mu \mathrm{g} / \mathrm{mL}$, as controls of bacterial inhibitors. The resultant mid-log phase cultures were diluted to the final concentration of $5 \times 10^{5} \mathrm{CFU} / \mathrm{mL}$, then $50 \mu \mathrm{L}$ was added to each well of the compound-containing 96-well plates (Corning; Cat. No. 3641, NBS), giving a final compound concentration range from $0.015 \mu \mathrm{g} / \mathrm{mL}$ to $32 \mu \mathrm{g} / \mathrm{mL}$ for control inhibitors and $32 \mu \mathrm{g} / \mathrm{mL}$ for test compounds. All the plates were covered and incubated at $37^{\circ} \mathrm{C}$ for $24 \mathrm{~h}$.

Inhibition of bacterial growth was determined visually and was recorded at $32 \mu \mathrm{g} / \mathrm{mL} ; 100 \%$ inhibition was identified.

\section{MIC assay}

The primary bacteria panel, including E. coli ATCC 25922, K. pneumoniae ATCC 700603 (MDR), A. baumannii ATCC 19606, P. aeruginosa ATCC 27853, and S. aureus ATCC 43300 (MRSA) were cultured in $\mathrm{MHB}$ at $37^{\circ} \mathrm{C}$ overnight. 


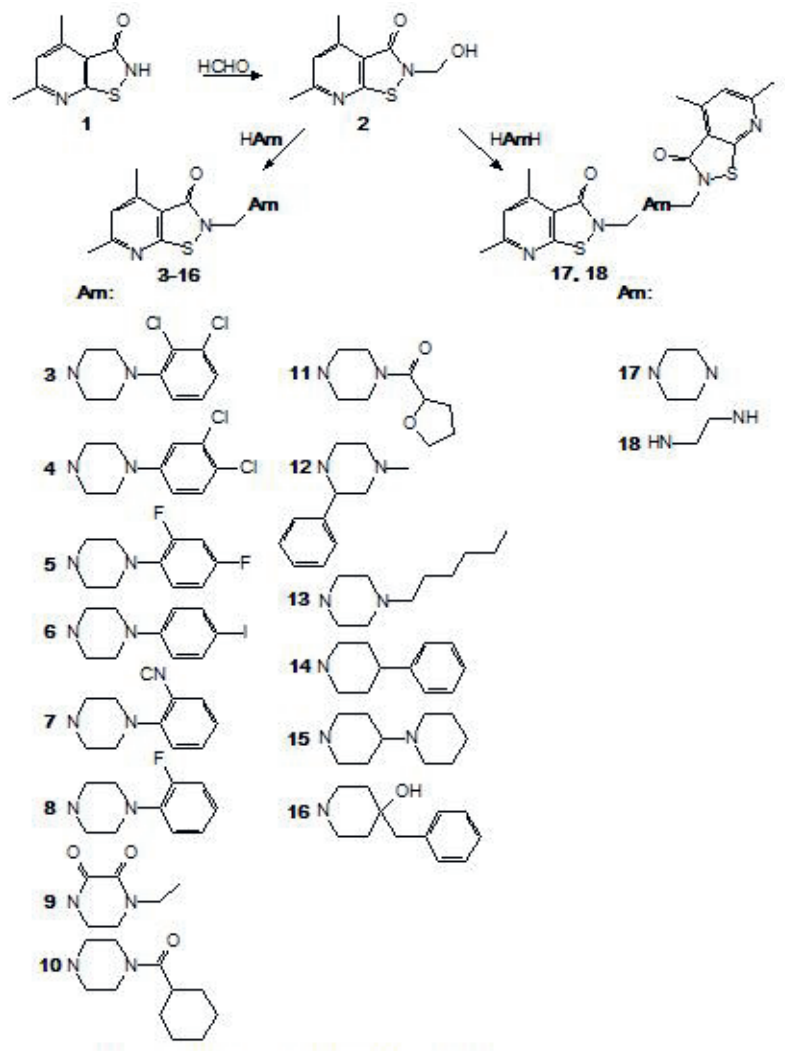

Fig. 1. Synthetic route of compounds 3-18

Table 1. Single point bacterial inhibition results

\begin{tabular}{|c|c|c|c|c|c|}
\hline Compound & $\begin{array}{l}\text { E.coli } \\
\text { ATCC } \\
25922\end{array}$ & $\begin{array}{c}\text { K. pneumoniae } \\
\text { ATCC } 700603 \\
\text { (MDR) }\end{array}$ & $\begin{array}{l}\text { A. baumannii } \\
\text { ATCC } 19606\end{array}$ & $\begin{array}{c}\text { P. aeruginosa } \\
\text { ATCC } 27853\end{array}$ & $\begin{array}{c}\text { S. aureus } \\
\text { ATCC } 43300 \\
\text { (MRSA) }\end{array}$ \\
\hline \multicolumn{6}{|c|}{ concentration $[\mu \mathrm{g} / \mathrm{mL}]$} \\
\hline 3 & $>32$ & $>32$ & $>32$ & $>32$ & $>32$ \\
\hline 4 & $>32$ & $>32$ & $>32$ & $>32$ & $>32$ \\
\hline 5 & $>32$ & $>32$ & $>32$ & $>32$ & $>32$ \\
\hline 6 & $>32$ & $>32$ & $>32$ & $>32$ & $>32$ \\
\hline 7 & $>32$ & $>32$ & $>32$ & $>32$ & $>32$ \\
\hline 8 & $>32$ & $>32$ & $>32$ & $>32$ & $>32$ \\
\hline 9 & $>32$ & $>32$ & $>32$ & $>32$ & $>32$ \\
\hline 10 & $>32$ & $>32$ & $>32$ & $>32$ & $>32$ \\
\hline 11 & $>32$ & $>32$ & $>32$ & $>32$ & $>32$ \\
\hline 12 & $>32$ & $>32$ & 32 & $>32$ & $>32$ \\
\hline 13 & $>32$ & $>32$ & 32 & $>32$ & 32 \\
\hline 14 & $>32$ & $>32$ & $>32$ & $>32$ & $>32$ \\
\hline 15 & $>32$ & $>32$ & $>32$ & $>32$ & $>32$ \\
\hline 16 & $>32$ & $>32$ & $>32$ & $>32$ & $>32$ \\
\hline 17 & $>32$ & $>32$ & $>32$ & $>32$ & $>32$ \\
\hline 18 & $>32$ & $>32$ & $>32$ & $>32$ & $>32$ \\
\hline Colistin & 0.06 & 0.03 & 0.06 & 0.25 & $>32$ \\
\hline Polymyxin B & 0.06 & 0.03 & 0.03 & 0.25 & $>32$ \\
\hline Vancomycin & $>32$ & $>32$ & $>32$ & $>32$ & 1 \\
\hline Daptomycin & $>32$ & $>32$ & $>32$ & $>32$ & 1 \\
\hline
\end{tabular}

MDR = multidrug resistance; $\mathrm{MRSA}=$ methicillin resistant Staphylococcus aureus.
A sample of each culture was then diluted 40 -fold in fresh $\mathrm{MHB}$ and incubated at $37^{\circ} \mathrm{C}$ for $1.5-3 \mathrm{~h}$. The compounds were serially diluted twice across the wells of non-binding surface 96-well plates (Corning; Cat. No. 3641, NBS), with compound concentrations ranging from $0.03 \mu \mathrm{g} / \mathrm{mL}$ to $64 \mu \mathrm{g} / \mathrm{mL}$, plated in duplicate. The resultant mid-log phase cultures were diluted to the final concentration of $5 \times 10^{5} \mathrm{CFU} / \mathrm{mL}$, then $50 \mu \mathrm{L}$ was added to each well of the compound-containing 96-well plates, giving a final compound concentration range from $0.015 \mu \mathrm{g} / \mathrm{mL}$ to $32 \mu \mathrm{g} / \mathrm{mL}$. All the plates were covered and incubated at $37^{\circ} \mathrm{C}$ for $24 \mathrm{~h}$.

Inhibition of bacterial growth was determined visually after $24 \mathrm{~h}$, where the MIC is recorded as the lowest compound concentration with no visible growth.

\section{Results}

The target dimethylisothiazolopyridine derivatives 3-18 were prepared by the Mannich reaction according to the general procedure shown in Fig. 1. The key intermediates: 4,6-dimethyl-3-oxo-2,3-dihydroisothiazolo[5,4-b]pyridine 1 and 2H-2-hydroxymethyl-4,6-dimethyl-3-oxo-2,3dihydroisothiazolo[5,4-b]pyridine 2 , were synthesized according to the method described recently. ${ }^{17}$ The preparation of the final isothiazolopyridines 3-18 involved the condensation of substrate 2 and the appropriate derivatives of secondary amine (piperazine, piperidine and ethylenediamine). Compound 8 was obtained earlier in another project. ${ }^{18}$ The reactions were carried out under modified conditions in relation to those described earlier. ${ }^{19} \mathrm{Un}$ der these conditions, most of the products were obtained with a high yield. The purity of the synthesized compounds was checked by elemental analyses. The structures of the isothiazolopyridines were determined based on spectral data analysis, such as IR and ${ }^{1} \mathrm{H}$ NMR.

The IR spectrum of compounds 3-18 showed a characteristic peak at 1650 $1680 \mathrm{~cm}^{-1}$ due to the carbonyl function of isothiazolopyridine in position 3 . In the case of compounds 9,10 and 11 , additional peaks of the carbonyl groups appeared.

The ${ }^{1} \mathrm{H}$ NMR spectra of compounds 3-16 displayed characteristic singlet signals derived from methyl groups of isothiazolopyridine appeared at $2.55-2.75 \mathrm{ppm}$ integrating for 3 protons. In the case of compounds 17 and 18, these signals had twice the integration corresponding to 6 protons. 
In the primary screen 2 compounds - 12 and $13-$ showed antimicrobial activity that could be considered a "primary hit", where a "primary hit" is defined as an MIC $\leq 32 \mu \mathrm{g} / \mathrm{mL}$. MIC values were determined for these compounds against the strains, where activity was seen in the primary screen. None of these compounds were considered suitable for further investigation following MIC confirmation, as the cut-off value was $\leq 16 \mu \mathrm{g} / \mathrm{mL}$, which none of the compounds possessed in these assays. A full set of results is presented in Table 1.

\section{Conclusions}

In order to develop novel antimicrobial compounds, a series of isothiazolopyridine derivatives was synthesized and characterized by physiochemical and spectral means. The synthesized compounds were evaluated for their antimicrobial potentials. From all the compounds, only compounds 12 and 13 were found to be effective against $A$. baumannii and $S$. aureus strains. However, these compounds showed weaker activity compared to the reference drugs.

It should be added that the low antibacterial activity of the compounds may be the result of insufficient solubility of the compounds under the conditions in which the tests were carried out.

\section{References}

1. Magiorakos AP, Srinivasan A, Carey RB, et al. Multidrug-resistant, extensively drug-resistant and pandrug-resistant bacteria: An international expert proposal for interim standard definitions for acquired resistance. Clin Microbiol Infect. 2012;18(3):268-281.

2. Alanis AJ. Resistance to antibiotics: Are we in the post-antibiotic era? Arch Med Res. 2005;36(6):697-705.

3. Ranft D, Lehwark-Yvetot G, Schaper K, Buge A. N1-Hetaryl substituted pyridine- and pyrazinecarboxamidrazones with antimycobacterial activity. Arch Pharm. 1997;330(6):169-172.

4. Carta A, Paglietti G, Rahgar Nikookar M, Sanna P, Sechi L, Zanetti S. Novel substituted quinoxaline 1,4-dioxides with in vitro antimycobacterial and anticandida activity. Eur J Med Chem. 2002;37(5):355-366.
5. Renau T, Sanchez J, Gage J, et al. Structure-activity relationships of the quinolone antibacterials against mycobacteria: Effect of structural changes at N-1 and C-7. J Med Chem. 1996;39(3):729-735.

6. Pagani G, Borgna P, Piersimoni C, Nista D, Terreni M, Pregnolato M. In vitro anti-Mycobacterium avium activity of $\mathrm{N}$-(2-hydroxyethyl)1,2-benzisothiazol-3(2H)-one and -thione carbamic esters. Arch Pharm. 1996;329:421-425.

7. Okachi R, Niino H, Kitaura $\mathrm{K}$, et al. Synthesis and antibacterial activity of 2,2'-dithiobis(benzamide) derivatives against Mycobacterium species. J Med Chem. 1985;28(12):1772-1779.

8. Ortega M, Montoya M, Jaso A, et al. Antimycobacterial activity of new quinoxaline-2-carbonitrile and quinoxaline-2-carbonitrile 1,4-di-Noxide derivatives. Pharmazie. 2001;56(3):205-207.

9. Zani F, Incerti M, Ferretti R, Vicini P. Hybrid molecules between benzenesulfonamides and active antimicrobial benzo[d]isothiazol3-ones. Eur J Med Chem. 2009;44(6):2741-2747.

10. Shimizu M, Shimazaki T, Yoshida T, Ando W, Konakahara T. Synthesis of 2-alkylidene-3,1-benzoxathiin-4-ones by the Pummerer type reaction. Tetrahedron. 2012;68:3932-3936.

11. Collier PJ, Ramsey AJ, Austin P, Gilbert PJ. Growth inhibitory and biocidal activity of some isothiazolone biocides. Appl Bacteriol. 1990; 69(4):569-577.

12. Fuller SJ, Denyer SP, Hugo WB, Pemberton D, Woodcock PM, Buckley AJ. The mode of action of I,2-benzisothiazolin-3-one on Staphylococcus aureus. Lett Appl Microbiol. 1985;1(1):13-15.

13. Collier PJ, Ramsey A, Waigh RD, Douglas KT, Austin P, Gilbert P. Chemical reactivity of some isothiazolone biocides. J Appl Bacteriol. 1990;69(4):578-584.

14. Brickner SJ, Hutchinson DK, Barychyn MR, et al. Synthesis and antibacterial activity of U-100592 and U-100766, two oxazolidinone antibacterial agents for the potential treatment of multidrugresistant gram-positive bacterial infections. J Med Chem. 1996; 39(3):673-679.

15. Barbachyn MR, Hutchinson DK, Brickner SJ, et al. Identification of a novel oxazolidinone (U-100480) with potent antimycobacterial activity. J Med Chem. 1996;39(3):680-685.

16. Blaskovich MA, Zuegg J, Elliott AG, Cooper MA. Helping chemists discover new antibiotics. ACS Infect Dis. 2015;1(7):285-287.

17. Malinka W, Rutkowska M. Synthesis and anorectic activity of $2 \mathrm{H}-4,6$-dimethyl-2-[(4- phenylpiperazin-1-yl)methyl]-3-oxo-2,3dihydroisothiazolo[5,4-b]pyridine. Farmaco. 1997;52(10):595-601.

18. Malinka W, Świątek P, Filipek B, Sapa J, Jezierska A, Koll A. Synthesis, analgesic activity and computational study of new isothiazolopyridines of Mannich base type. Farmaco. 2005;60(11-12): 961-968.

19. Malinka W, Karczmarzyk Z, Sieklucka-Dziuba M, Sadowski M, Kleinrok Z. Synthesis and in vivo pharmacology of new derivatives of isothiazolo[5,4-b]pyridine of Mannich base type. Farmaco. 2001;56(12):905-918. 